\title{
Effect of bedside teaching activities on patients' experiences at an Ethiopian hospital
}

\author{
F A Gebrekirkos, ${ }^{1} \mathrm{MD}$, FCS-COSECSA; J M van Wyk, ${ }^{2} \mathrm{BSc}$ Ed, MEd, PhD
}

${ }^{1}$ Department of Surgery, College of Health Sciences, and Center for Health Professions Education, Mekelle University Hospital, Ethiopia

${ }^{2}$ Clinical and Professional Practice, Nelson R Mandela School of Medicine, College of Health Sciences, University of KwaZulu-Natal, Durban, South Africa

Corresponding author: JMvan Wyk (vanwykj2@ukzn.ac.za)

Background. Clinical bedside teaching is more effective when done at the bedside. The number of medical schools in Ethiopia has increased tenfold in 8 years to meet the Millennium Development Goals. The increased number of students at the patient's bedside has been met with mixed feelings by patients.

Objective. To determine patients' perceptions of bedside teaching during their admission to the medical and surgical wards at Mekelle University Hospital, Ethiopia.

Methods. A 32-item questionnaire was used for data collection. Patients used a Likert scale to rate their perceptions of the quality of their hospital stay with regard to teaching, clerkships and physical examinations. Items where respondents scored less than the median of 67 (interquartile range 21) were categorised as displaying a negative attitude.

Results. Patients (60\%) did not favour the bedside teaching activities. No significant association was found with age, sex, occupation, literacy level, duration of hospital stay, and ward. Patients $(>80 \%)$ also did not understand the discussions following teaching sessions, and claimed to be unaware of the teaching status of the hospital. Patients $(>80 \%)$ did not understand the role of the students and were anxious when left alone to be examined and clerked by them. Conclusion. The rights of patients in medical education should be emphasised. Patients should be informed about the role of students at teaching hospitals and about their rights and responsibilities as patients. Institutional protocols and country-wide guidelines can help to regulate the number of times that a patient should be clerked and physically examined by students. Instructors should ensure that patients understand the purpose of the discussion that follows the examination.

Afr J Health Professions Educ 2016;8(2):144-147. DOI:10.7196/AJHPE.2016.v8i2.520

Clinical teaching, including interpersonal and communication skills, has been known to be more effective when conducted at the bedside. ${ }^{[1,2]}$ Osler $^{[3]}$ considered learning in the presence of a patient of the utmost importance, and stated it as a rule to have 'no teaching without a patient for a text, and the best teaching to consist of lessons taught by the patient himself'.

Bedside teaching involves a process where leaners use their senses hearing, vision, smell and touch - to learn about the patient ${ }^{[4]}$ to enhance their humanistic skills, which are often neglected in classroom settings. ${ }^{[5]}$ The process requires students to spend unsupervised time with patients, from whom they take histories and on whom they perform physical examinations independently. ${ }^{[1]}$ The development of medical students' clinical competence is a long process and the use of live patients for teaching during this process poses valid ethical concerns with regard to the patient's welfare.

A number of studies have been conducted on the effect of bedside activities on patients. ${ }^{[6-11]}$ Some studies explored patients' perceptions of having students present during bedside teaching sessions; ${ }^{[9]}$ some investigated their perceptions on the effect of bedside v. conference room presentations; ${ }^{[12]}$ while other studies obtained patients' views on the ability of students to conduct interviews. ${ }^{[7]}$ Most studies reported that patients were tolerant towards bedside teaching activities and generally positive towards students. ${ }^{[2,6,8,10,12]}$ However, there was resistance from patients who did not perceive any benefit from being part of the teaching process. ${ }^{[6]}$ Cooke et al.'s ${ }^{[6]}$ study reported that differences in patients' tolerance levels and perceptions are linked to their level of education, i.e. implying that better-educated patients were more tolerant. These authors also reported that differences in patients' reactions towards bedside teaching activities depended on the patient's race.
The situation in Ethiopia is new and needs special attention. Driven by the desperate shortage of health personnel and a need to reach the Millennium Development Goals, the Ethiopian Ministry of Health has approved a plan to improve the training of medical and healthcare professionals. This decision led to a drastic increase in training facilities, i.e. from 3 to 32 medical schools in the past 8 years, 438 newly qualified medical doctors per year compared with 90 a decade ago, and the admission of $6000-6500$ students in 2011. ${ }^{[13]}$ The increased enrolment of students has manifested in much higher ratios of students to medical staff at the patient's bedside. Anecdotal evidence suggests that patients have mixed feelings about the increased presence of students at their bedside, and to date there has not been any research to explore the patient's view and experience in the context of an Ethiopian teaching hospital. This gap is being addressed by the current study.

The increased student-to-patient ratio has many consequences. Firstly, it impacts on clinical teaching and students' learning, as they are unlikely to gain adequate experience in the absence of sufficient patients to clerk and perform physical examinations. Secondly, the disproportionate ratios have an effect on teaching, as it is becoming increasingly more difficult to teach students how to perform physical examinations correctly and to detect physical findings on fewer patients. Patients who are already ill and experiencing discomfort find it annoying and tiring to be clerked repeatedly by different students from various academic years, which undoubtedly has an effect on their assessment of the clinical competence of the students. ${ }^{[10]}$

Ethiopia lacks a standardised approach to guide training institutions on an appropriate or approved minimum number of students allowed per patient. It is believed that such a guide would ensure that the patients' treatment and the students' learning remain optimal. An example of a 
policy to manage such a process is found in the context of India. An Indian admission policy allocates 100 students to a 500-bed healthcare facility, or 200 students for 900 beds per year in facilities with a bed occupancy rate of $75 \%$. This proportion excludes beds reserved for subspecialties. ${ }^{[14]}$

Ethiopia, however, does not have an established standard to guide the allocation of students in the medical wards. Given the lack of guidelines on the ideal number of students to allocate to the bedside in the African context and the centrality of the patient in the training of clinical competences, our study explored the perceptions and attitudes of patients towards the clinical teaching process.

\section{Methods}

This quantitative, cross-sectional study was conducted in October 2013. A 32-item questionnaire (Appendix 1) was used to collect data from adult patients $(N=256)$ admitted to the medical and surgical wards during that period. Patients were asked to respond to questions enquiring about the effect of bedside teaching activities, student clerkships and physical examinations on their experience in hospital and the quality of their hospital stay.

The self-developed questionnaire was designed in English and translated into Amharic and Tigrigna - the latter is the language spoken by the majority of the people in this region of Ethiopia. In the absence of a validated, reliable data collection tool, we developed a questionnaire based on the literature around a number of focal areas of studies on patients' perceptions of clinical teaching. ${ }^{[9,10]}$ For example, statements such as 'I have enjoyed bedside teaching,' 'I felt anxious when clerked by students', and 'I have been told that students will be spending some time with me' were extrapolated from Nair et al.'s ${ }^{[9]}$ study. Statements such as 'Patients understood what was being discussed', and 'Comfortable that their case was being discussed' were obtained from Seo et al'.s ${ }^{[12]}$ study.

The focal areas included questions exploring patients' awareness and perceptions of the role of Mekelle University Hospital as a medical training facility; their perceptions of the discussions during bedside teaching about their illness and the presence of students around them; their level of comfort and/or anxiety at being clerked by unsupervised students; their tolerance and participation in responding to students' questions; and their perceptions of benefiting and gaining an improved understanding of their illness owing to being treated at a teaching hospital.

Patients were asked to indicate their responses to each item on a 5-point Likert scale. The questionnaires were distributed by ward nurses, who also assisted illiterate patients.

Data were captured on an Excel spreadsheet. The responses were computed using the Statistical Package for the Social Sciences 16 (SPSS 16) (SPSS Inc., USA). The categories 'strongly agree' and 'agree' were aggregated to indicate a positive score, and the 'strongly disagree' and 'disagree' were aggregated to obtain a negative score. Descriptive statistics were computed on each item. Items where respondents scored less than the median of 67 (interquartile range (IQR) 21) were categorised as displaying a negative attitude. The median was used because the mean and standard deviation were found to be 72.0236 and 14.49 , respectively. The frequency distribution graph was positively skewed (skewness 1.253 ).

Ethical approval for the study was obtained from the Health Research Ethics Review Committee of the College of Health Sciences, Mekelle University, Ethiopia. All the patients who participated in the study signed an informed consent document. A copy of the questionnaire is available from the first author.

\section{Results}

A total of 150 questionnaires were distributed, of which 127 (84.7\%) were completed and returned. The completed questionnaires were received from 74 and 53 patients who had been admitted to the internal medicine and surgical wards, respectively, during October 2013.

Eighty-four (61.0\%) of the 127 respondents were male. Their ages ranged from 16 to 84 years. One hundred and twenty (94.4\%) respondents spoke Tigrigna, the local language. Forty-seven (37.0\%) respondents were illiterate at the time of the study, 30 (23.6\%) had attended only elementary school, $29(22.8 \%)$ had attended high school, and 21 (16.5\%) had attended a higher education institution.

Eighty-seven percent $(n=127)$ did not understand the discussions during the bedside teaching sessions. Before hospitalisation, nearly $87.0 \%(n=110)$ were not aware that students would be spending time with them and claimed not to understand the role of the students in the ward. Eighty-one percent $(n=103)$ did not see any benefit to being treated at a teaching hospital. Eighty-five percent $(n=108)$ of the respondents did not at all understand what was being discussed during the bedside teaching session.

Patients (86.6\%; $n=110)$ indicated feeling uncomfortable in the presence of unsupervised students; $81.1 \%$ ( $n=103)$ felt anxious when left alone with students for examination and clerking; and $82.2 \%(n=104)$ did not like having students around them. Seventy-nine percent $(n=100)$ felt uncomfortable when being clerked by students.

Respondents $(70.1 \% ; n=89)$ thought that bedside teaching may result in a breach of confidentiality, and $65.0 \%(n=83)$ were unwilling (would not volunteer) to have their case discussed at the bedside teaching session. Only $33.0 \%(n=42)$ thought that bedside teaching sessions had assisted them in gaining a better understanding of their illness. Sixteen percent were aware that they were being treated at a teaching hospital.

In response to the question that explored the patients' awareness of and attitude towards bedside teaching activities, $48.0 \%(n=61)$ were not in favour of such activities, and $48.0 \%$ had an attitude score below the median of 67 .

No significant association was found with age, sex, occupation, address (urban v. rural), literacy level, duration of hospital stay, or the wards to which the patients had been admitted.

\section{Discussion}

The ultimate goal of medical education is to improve patient care. This study explored the perceptions of adult patients towards students in the context of an increased student presence in the ward during bedside teaching activities, the patients' perceptions of the benefit of admission to a teaching hospital, and their role in the training of student doctors. While bedside teaching was meant to be beneficial to learners and patients, findings suggest that patients felt anxious when approached by unsupervised students and that they were ill informed of the reasons for and details of the bedside conversations between the teacher and the student. Patients' reluctance to having their case discussed during bedside sessions also stemmed from concerns about confidentiality.

These findings are similar to those of a study in Oxford, UK, where patients expressed reservations about being left in the care of student doctors. ${ }^{[6]}$ Illiterate patients and those who were not well informed of the reasons for having students at the bedside, were more anxious. A study in Belgium similarly cautions clinicians not to increase dosages of hypertensive medication of patients in teaching hospitals, as blood pressure levels of patients became elevated when they were in the presence of student doctors. ${ }^{[15]}$ 
Patient care also includes the patient's mental perception of receiving appropriate care. Patients at this medical teaching hospital were sceptical of the facility and considered the high student presence untrustworthy. In this regard, the institution has failed to address the psychological needs of the patients.

It is obvious that patients need reassurance and that communication should be improved to ensure that bedside teaching sessions become an informative and enjoyable experience to them. Senior members of staff will have to play a more supportive role in explaining to patients that their health and participation in the teaching and learning processes are valued and their wellbeing and care are central in the bedside learning process. This lesson is reinforced in suggestions for good practice, requiring teachers to refrain from discussing embarrassing details in the presence of their patients and to display exemplary professional behaviour. ${ }^{[5]}$

Some of the ways to convey this message include ensuring that patients leave with a better understanding of their illnesses. It will reassure them and help them to see the benefit of having learned from the discussion of their case. It is also possible that a difference in the language of learning and that of the patient could lead to a misunderstanding in communication. This may have an effect on patients experiencing the bedside teaching sessions as tedious, especially in areas such as ours where the patients' education levels require more effort in relaying the purpose, process and outcome of the bedside examination.

Patients should be informed that they are being admitted to a teaching hospital and that it involves some degree of interaction with students who are being trained at the facility. An agreement, perhaps in the form of signed informed consent, may be administered on admission to ensure that patients are informed and agreeable to interact with students. The role of the students and the rights and responsibilities of patients should be better explained. Emphasis on this process will address patients' fears relating to confidentiality, or of being managed by students or incompetent persons.

Patients can benefit from being educated about the advantages of being treated in a teaching hospital and the value of their contribution in the teaching of future doctors. Students' attitudes towards patients should be monitored to ensure that the former create an environment of trust and professionalism in their interaction with patients. They should display honesty, integrity and confidentiality; clearly communicate their expectations about their capacity and limitations; and explain that they are there to learn and help in every possible way. Teachers should instruct students about demonstrating the aforementioned values to co-operate with patients, and to take their time to offer a full explanation of the disease.

The rights of patients in medical education should be emphasised. After each teaching session instructors should explain what they were discussing so that patients understand the purpose of the discussion. As almost all patients do not understand the medium of instruction (English), it will have a significant effect on patients' perceptions of the teaching activities if we can summarise and explain in their own language the proceedings and possible outcomes of the discussion.
Anecdotal evidence has shown that patient fatigue is definitely a factor in the way they experience their stay at our hospital. The perception of being treated unfairly is enhanced in the absence of clear guidelines on a reasonable number of students allowed per patient for teaching purposes. This can be resolved when medical educators, community stakeholders and the Ministry of Health collaborate to formulate regulations and guidelines for safe practices in training institutions.

Study limitations include the design, as this was an explorative quantitative study. Some issues to enhance our understanding should be explored more qualitatively. Furthermore, the study has been conducted in one region of the country, where a specific language is spoken and where the patients' education levels are relatively low. We therefore caution that results may not be generalised, but that educators compare their settings to ours and learn from the lessons that we have highlighted. The success of a patient education campaign and effect of the suggested recommendations will be monitored and reported.

\section{Conclusion}

Bedside teaching affords students the opportunity to understand the context of medicine better and to learn from the clinician as a role-model. It is important to note that it can be a tedious and stressful experience for patients and that those who are poorly informed will have a negative attitude towards the student and be less perceptive to the potential benefit of being part of the learning process. Every effort should be made to educate patients about the benefits of visiting a teaching hospital. The teaching institution should also regulate the process to ensure patient confidentiality, comfort and safety, while senior members of staff should become more visible for monitoring and role-modelling.

\section{References}

1. Bell K, Boshuizen HPA, Scherpbier AJJA, Dornan TL. When only the real thing will do: Junior medical students learning from real patients. Med Educ 2009;43(11):1036-1043. DOI:10.1111/j.1365-2923.2009.03508.

2. Olson LG, Hill SR, Newby DA. Barriers to student access to patients in a group of teaching hospitals. Med Austr 2005;183(9):461. https://www.mja.com.au/system/files/issues/183_09_071105/ols10328_fm.pdf (accesse Austr 2005;183(9):

3. Osler W. Aequanimitas; with other addresses to medical students, nurses and practitioners of medicine. Am J Med Sci 1905;130(2):337

4. Bekele A, Reissig D, Loffler S, Hinz A. Experiences with dissection courses in human anatomy: A comparison between Germany and Ethopia. Ann Anat 2011;193(2):163-167. DOI:10.1016/j.aanat.2010.10.007 5. Ramani S. Twelve tips to improve bedside teaching. Med Teach 2003;25(2):112-115. DOI:10.1080/0 142159031000092463

6. Cooke F, Galasko G, Ramrakha V, Richards D, Rose A, Watkins J. Medical students in general practice: How do patients feel? Br J Gen Pract 1996;46(407):361-362.

7. Feletti G, Carney S. Evaluating patients' satisfaction with medical students' interviewing skills. Med Educ 1984;18(1):15-20. DOI:10.1111//.1365-2923.1984.tb01470.x

8. Lehmann LS, Brancati FL, Chen M-C, Roter D, Dobs AS. The effect of bedside case presentations on patients ${ }^{3}$ perceptions of their medical care. N Engl J Med 1997;336(16):1150-1156. DOI:10.1056/NEJM199704173361606 Nair B, Coughlan J, Hensley M. Student and patient perspectives on bedside teaching. Med Educ 1997;31(5):341346. DOI:10.1046/j.1365-2923.1997.00673.x

10. Nair B, Coughlan J, Hensley M. Impediments to bed-side teaching. Med Educ 1998;32(2):159-162. DOI:10.1046 j.1365-2923.1998.00185.x

11. Spencer J, Blackmore D, Heard S, et al. Patient-oriented learning: A review of the role of the patient in the education of medical students. Med Educ 2000;34(10):851-857. DOI:10.1046/j.1365-2923.2000.00779.x

12. Seo M, Tamura K, Morioka E, Shijo H. Impact of medical rounds on patients' and residents' perceptions at a university hospital in Japan. Med Educ 2000;34(5):409-411. DOI:10.1046/j.1365-2923.2000.00516.x

13. Zhao F. Health Resources for Health: The Ethiopian Success Story. Ethiopia: Human Development AFDB, 2012

14. Rao M, Rao K, Kumar A, Chatterjee M, Sundararaman T. Human resources for health in India. Lancet 2011;377(9765):587-598. DOI:10.1016/S0140-6736(10)61888-0

15. Matthys JH. Teaching students in general practice can affect patients' blood pressure. BMJ 2005;331(7513):406. DOI:10.1136/bmj.331.7513.406-b 


\section{Research}

Appendix 1. Results of the questionnaire

\begin{tabular}{|c|c|c|c|c|c|}
\hline Variable & Strongly agree & Agree & $\begin{array}{l}\text { Never thought } \\
\text { about it }\end{array}$ & Disagree & $\begin{array}{l}\text { Strongly } \\
\text { disagree }\end{array}$ \\
\hline The role of the students is clearly communicated to me & 6 & 16 & & 102 & 3 \\
\hline I think there is a benefit to being treated at a teaching hospital & 8 & 13 & 3 & 100 & 3 \\
\hline I was told/informed that this is a teaching hospital & 6 & 7 & 7 & 102 & 5 \\
\hline I am aware that students will be spending time with me & 5 & 9 & 8 & 99 & 6 \\
\hline $\begin{array}{l}\text { I clearly understand that my case will be discussed with students and } \\
\text { their teachers }\end{array}$ & 7 & 14 & 4 & 100 & 2 \\
\hline I do not know what the students are talking about at the bedside & 8 & 100 & 6 & 11 & 2 \\
\hline I feel uncomfortable with the presence of students around me & 13 & 97 & 5 & 10 & 2 \\
\hline I like it when there are students around me & 3 & 15 & 4 & 101 & 4 \\
\hline I feel anxious when students and their teachers are talking about me & 11 & 60 & 11 & 35 & 10 \\
\hline I believe students' involvement can improve my clinical outcome & 12 & 85 & 3 & 22 & 5 \\
\hline I don't have any clue what the discussion is about in the bedside teachings & 17 & 96 & 2 & 10 & 2 \\
\hline Bedside teaching has helped me to understand my illness better & 9 & 29 & 4 & 81 & 4 \\
\hline I have enjoyed being used as an example during bedside teaching & 9 & 34 & 6 & 73 & 5 \\
\hline I think that bedside teaching will lead to a breach of confidentiality & 11 & 78 & 15 & 20 & 3 \\
\hline I can tell the difference between students, instructors, and ward nurses & 17 & 58 & 14 & 29 & 9 \\
\hline I think that I am being managed by students & 6 & 64 & 12 & 33 & 12 \\
\hline I find it uncomfortable when students are checking up on (clerking) me & 12 & 88 & 5 & 19 & 3 \\
\hline I feel anxious when students visit me without the presence of the instructor & 9 & 94 & 2 & 17 & 5 \\
\hline I like it when young students come and clerk me & 7 & 24 & 5 & 85 & 6 \\
\hline I find it painful when students are doing a physical examination on me & 10 & 74 & 11 & 25 & 7 \\
\hline Students have good clinical knowledge & 6 & 35 & 11 & 70 & 5 \\
\hline I will volunteer to be clerked and examined by students next time & 10 & 32 & 2 & 78 & 5 \\
\hline I think I am unnecessarily being asked about my health many times & 7 & 91 & 10 & 18 & 1 \\
\hline $\begin{array}{l}\text { I am being asked about my health because the questions are very } \\
\text { important for my condition }\end{array}$ & 15 & 30 & 20 & 62 & - \\
\hline My body part is unnecessarily being touched too many times & 13 & 89 & 7 & 12 & 6 \\
\hline Medical students take my time unnecessarily & 11 & 84 & 6 & 22 & 4 \\
\hline The discussion in bedside teaching takes my time unnecessarily & 13 & 78 & 8 & 22 & 6 \\
\hline
\end{tabular}

